\title{
Autonomie als strebendes Konzept
}

- Ein multidisziplinärer Ansatz -

Die verfassungsrechtlich vorgegebene Unabhängigkeit der Dritten Gewalt schließt für ihre uneingeschränkte Entfaltung notwendig auch die Eigenverantwortung für den gesamten Organisations- und richterlichen Personalbereich ein (mit Ausnahme der Richterwahl). Eine Gesamtbetrachtung unter Zusammenführung der organisationssoziologisch, anthropologisch und psychologisch wirkenden Erkenntnisse aus Soziologie, Organisationswissenschaften, Betriebs- und Volkswirtschaft sowie nicht zuletzt Evolutionsbiologie und Neurologie führt zu dem Ergebnis, dass größtmögliche Ausdifferenzierung von Eigenverantwortung bis zur individuellen und kleinsten Einheitsebene zur größtmöglichen Effizienz und Qualität führt. Die Orientierung an der Aufgabenstellung, hier der Rechtsprechung, ist der allein gültige Maßstab.

\section{Aufgabenstellung und Situation}

Die rechtsprechende Tätigkeit hat in den letzten drei Jahrzehnten einen tief greifenden Wandel erfahren. Sie ist schwieriger, zeitaufwendiger und komplexer geworden. Ursachen sind die nicht nur durch neue Kommunikations- und Arbeitstechniken bedingten, ungeheuer schnell sich wandelnden wirtschaftlichen, sozialen, finanziellen und gesellschaftlichen Verhältnisse. Die Schnelligkeit des Wandels und die Durchdringung aller Lebens- und Wirtschaftsbereiche überfordern die politischen und gesellschaftlichen Instanzen. Die sozio-politische Folge ist die rechtsstaatlich wohl unvermeidlich wachsende Zuweisung gesellschaftlicher und ökonomischer Problemfälle an die Gerichte aufgrund der immer deutlicheren Tendenz, alle Probleme dieser Gesellschaft in Rechtsprobleme zu transponieren. Das Transponieren dieser Problemfälle in eine einer rechtlichen Lösung zugangsfähigen Konstellation tatbestandlicher und rechtlicher Bedingtund Abhängigkeiten im Rahmen eines kommunikativen Prozesses ist die hoch komplexe Aufgabe der Rechtsprechung. Bei der Erfüllung dieser Aufgabe kann die das Rechtsproblem rahmende soziale Wirklichkeit zu keiner Zeit von den Richterinnen und Richtern ausgeschaltet werden. ${ }^{1}$ Das heißt, sie haben in jedem zur Entscheidung anstehenden Einzelfall Kohärenz von Sozial- und Rechtsordnung herzustellen: Rechtsprechung als hochkomplexer, heute mehr denn je sich ständig wandelnden Anforderungen unterworfener Prozess.

\section{Rahmenbedingungen}

Dieser Prozess wird wesentlich beeinflusst von den organisatorischen und personellen Rahmenbedingungen. Die Gestaltung und Gestaltbarkeit der Bedingungen ist abhängig

1 Stegmaier, Wissen, was Recht ist, 2009, S. 393. 
von den Vorgaben, die die Fähigkeit und Bereitschaft der Betroffenen sich dieser, die eigentliche Aufgabe der Rechtsprechung rahmenden Verantwortung zu stellen, zu fördern im Stande sind. Zur Ermittlung der Vorgaben ist zurückzugreifen auf die Erfahrungen und Erkenntnisse aus unterschiedlichen Wissenschafts- und Organisationsbereichen.

\section{Das Prinzip der Subsidiarität}

$\mathrm{Zu}$ beginnen ist mit der Aufnahme- und Verarbeitungsbereitschaft von Informationen unterschiedlichster Art. Diese Bereitschaft wächst mit zunehmender Primärkompetenz. Informationsrezeption wirkt immer dann kompetenz- und auch persönlichkeitssteigernd, wenn sie in Entscheidungszusammenhänge eingebunden ist. Das heißt organisationseffizient, Sach- ebenso wie Organisationsentscheidungen fallen umso funktionsgerechter aus, je näher die mit der Entscheidung Befassten der zu beurteilenden Sachlage stehen und je mehr sich diese der eigenen unmittelbaren Verantwortung bewusst sind; ein gegenseitig und voneinander abhängig wirkender Prozess. Diese Erkenntnis trifft sich mit der objektiv feststellbaren Entwicklung, dass bei zunehmender Komplexität von organisatorischen Einheiten die Bedeutung zentraler Einheiten ständig abnimmt, während die Funktionskompetenz der dezentralen Einheiten (vor Ort) im gleichen Maße wächst. Je komplexer die Verhältnisse, desto mehr kommt es auf die Teile an. Dies gilt gerade und insbesondere für die Organisation der Rechtsprechung und ihre Gliederung in Gerichtsbarkeiten und Gerichte verschiedener Größen und Instanzen. Jedes Amtsgericht ist im Rahmen seiner regionalen Zuständigkeit nicht nur der Entscheidung im einzelnen Rechtsstreit am nächsten, sondern auch der Organisationsaufgabe und verfassungskonformen Organisationsverantwortung bezogen auf den Rechtsprechungsprozess.

Der technisch und funktional unumkehrbare Prozess zur Dezentralisierung organisatorischer Abläufe findet seine Bestätigung im Themenbereich Globalisierung, Regionalisierung, Ablehnung von Fremdsteuerung sowie Rückbesinnung auf lokale wie regionale Stärken und Werte. Dank Autonomie kleinster Einheiten können intelligente Komponenten die Ziele ihres eigenen Kreises besser lösen aufgrund von Kenntnissen und Identitäten vor Ort und können sich dann mit größeren Gruppen zusammenschließen, um ihre intelligenten Fähigkeiten zu vereinigen.

\section{Anleihe aus der Evolutionsbiologie}

Die Entwicklung geht einher mit Erkenntnissen aus der Evolutionsbiologie: Lebensfähige Systeme organisieren sich weitgehend selbst. Das System selber ist der Akteur. Jeder biologische Organismus antwortet auf die Umwelt, und zwar unmittelbar und nicht weitgehend denaturiert und mediatisiert durch externe „organisatorische Verschaltungen". Selbstsozialisierung infolge kooperativen Verhaltens ist das Lebensprinzip. Bezogen auf die Justiz bedeutet dies Eigenverantwortung der im System Agierenden und Kooperierenden, nicht gesteuert durch externe Vorgaben, sondern durch die Herstellung der Identität von Ort, Zeit und Handlung. Diese Identität ist gleichzeitig auch immer 
maßgebend für jede Gestaltung im Ablauf eines Rechtsprechungsprozesses, in dessen Zentrum sich als Interaktionsraum die mündliche Verhandlung befindet.

\section{Anleihe aus der Neurologie}

Nahezu zwangsläufig erläutern die in den letzten zehn Jahren gewonnenen Erkenntnisse der Neurologie die unverzichtbare Notwendigkeit von eigenverantwortlichem Handeln z. B. in einem System wie jenem der Rechtsprechung. Die Neurologie beschreibt mit dem Begriff der Plastizität das Phänomen, wie sich das menschliche Gehirn selbst organisiert, indem sich Funktion und Struktur lebenslänglich gegenseitig so beeinflussen, dass beide sich im positiven Sinne weiterentwickeln können; jedoch nur in stets lebendigen Prozessen, das heißt, wenn fortwährend entsprechende Provokationen von Struktur und Funktion erfolgen. Dies kann für die Frage nach dem Funktionieren von Systemen, wie z.B. Rechtsprechung, bedeuten, dass durch die selbstverantwortliche Wahrnehmung aller entscheidenden Organisations- und Kommunikationsaufgaben Richterinnen und Richter ein Berufsleben lang auf die Funktion und Strukturierung von Rechtsprechung und ihren Aufgaben im positiv weiterentwickelnden und gestaltenden Sinne einwirken. Hervorzuheben ist bei dem Wirkungsmuster von „Plastizität“ das sich bei Rechtsprechungs-Kausalzusammenhängen zwangsläufig einstellende Ergebnis, dass Qualifikations- und Kompetenzerweiterungen für die Entscheidung im Einzelfall ebenso entstehen wie für die Gestaltung der Gesamtaufgabe „Rechtsprechung“. Das System wird von nachhaltiger Entwicklung getragen. In jedem Stadium des Rechtsprechungsprozesses kann so das Bearbeitete auf die Bearbeitenden zurückwirken und die Orientierung an der System- und damit der Lebens- wie Gesellschaftsdienlichkeit verstärken. Denn nur im individuellen, eigenverantwortlichen Tun liegt die Chance zu erfahren, was Rechtsprechung im Sinne des gesellschaftlichen Auftrags braucht.

\section{Interaktive Rechtsprechung - ein strebendes Konzept}

So trifft die für jede Rechtsprechungsentscheidung maßgebende, immer neu herzustellende Kohärenz von Sozial- und Rechtsordnung und letztlich auch Organisationsordnung auf günstige Voraussetzungen: Die mit der Rechtsprechung Beauftragten profitieren und lernen in allen Tätigkeitsbereichen von der gegenseitigen Beeinflussung bei der Besorgung von Organisationsfragen. Die tätigkeitsabhängige Kompetenz und Qualifikation aufgrund persönlicher Erfahrungen der in jedem Stadium eigenverantwortlich handelnden Richterinnen und Richter werden nutzbar für die Rechtsprechung. Zugleich profitiert und entwickelt sich die Organisation der Rechtsprechung selbst in ihren ordnenden und lenkenden Strukturen. Das heißt: Fortwährend neue Möglichkeiten von Effizienz- und Qualitätsgewinnen in allen Bereichen - ein strebendes Konzept.

Am Ende wird sich die Frage nach der persönlichen und sachlichen Unabhängigkeit der in solchen Systemen eigenverantwortlich, interaktiv Handelnden, nur gebunden durch die Gesetze und durch die im Rahmen ihrer eigenen Zuständigkeiten zugunsten des Systemzwecks selbst definierten Aktionsregeln, jenseits der insoweit bemühten gesetzlichen Regelungsabsichten de facto als eine rhetorische Frage erweisen. 